\title{
COMMUNICATIONS
}

\section{SURGICAL CLASSIFICATION OF SQUINTS WITH A VERTICAL DEVIATION*}

\author{
BY \\ ALFREDO VILLASECA \\ Hospital Salvador, Santiago, Chile
}

VERTICAL squints, either pure or associated with horizontal squints, are of great practical importance, since binocular single vision can only be attained if there is perfect parallelism of the visual axis in both the horizontal and vertical directions.

The statistics given by various authors show that vertical deviations are very frequent:

White and Brown (1939) found the following distribution in 1,062 cases : uncomplicated lateral deviation in 347 patients, uncomplicated vertical deviation in 358 , and combined vertical and lateral deviation in 357 .

Dunnington and Regan (1950) found that 50 per cent. of 79 cases of concomitant convergent strabismus showed a vertical component.

Scobee (1951), in 457 cases of convergent strabismus, found 195 (43 per cent.) with a vertical component. In his patients the frequency of involvement (paresis) of the vertically acting muscles was as follows:

Muscle No. of Cases

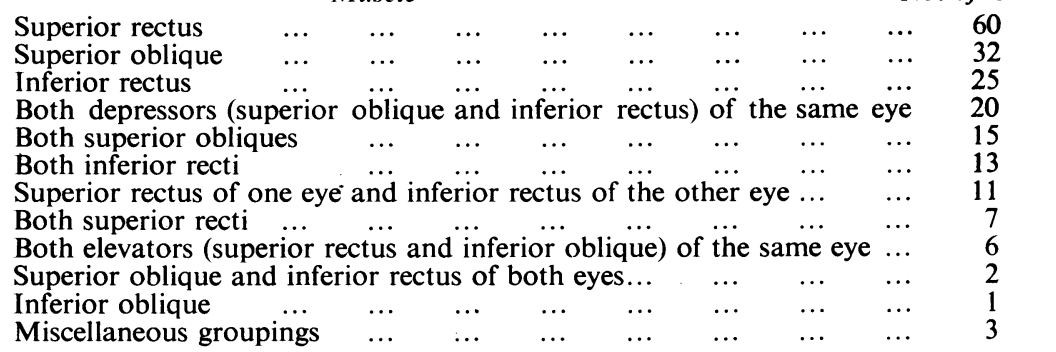

He makes no specific mention of patients with concomitant hypertropia or dissociated vertical divergence.

Bielschowsky (1938) classifies the disorders of the vertical motor muscles in five groups:

(1) pure concomitant vertical deviations;

(2) vertical deviations of paretic origin;

(3) deviations that show the features of unilateral or bilateral overfunction of the inferior oblique muscle;

(4) dissociated vertical deviations;

(5) vertical deviations showing the combined characteristics of several of the other groups.

*Received for publication October 8, 1954. 
(1) The angle of squint is independent of the direction of the gaze and of the transition of the fixation from one eye to the other.

(2) The vertical deviation increases in the field of action of the paralysed muscle and when the affected eye fixes.

(3) The primary spasm of the inferior oblique muscle is differentiated from the secondary contracture following a paralysis of the ipsilateral superior oblique or of the contralateral superior rectus. A differential symptom is the absence of a vertical deviation in the primary position of the eyes in cases of pure spasm, as opposed to the post-paretic cases where the hypertropia is also evident in the primary position. On testing the fields of fixation of either eye, it is seen that each field is considerably enlarged in the upper nasal quadrant in patients with primary spasm of both inferior obliques, without restriction in the upper temporal quadrant.

(4) Disorders of the vertical motor muscles known as dissociated vertical divergence, alternating hyperphoria, or double hyperphoria.

The separation of patients with over-function of the inferior oblique muscles from the second group with deviations of paretic origin, seems somewhat academic, and not well justified in Bielschowsky's classification. We nevertheless accept the existence of a pure spasm of the inferior oblique muscles (without paresis of the ipsilateral antagonist or contralateral synergist), as is typically manifested by the synkinetic overaction of the inferior oblique muscles in convergent squints due to overconvergence.

Confusion may arise in dealing with various types of vertical deviations and their different combinations with horizontal deviations. For this reason the following practical classification has been devised. In the first place vertical squints may be divided into two groups: (A) Primary, and (B) Secondary.

\section{(A) Primary Vertical SQuint}

These are independent of any horizontal deviation, and may be either isolated or accompanied by an added horizontal deviation. In fact, if binocular single vision is broken by a vertical deviation, there is no obstacle to prevent a lateral deviation of the eyes, which will generally become manifest as a convergent squint since convergence is overactive in childhood. If an exophoria existed previously, the horizontal deviation will be divergent.

\section{Group 1. Paresis of an Elevator or Depressor Muscle of One Eye.}

(i) The muscles more often affected are the superior rectus or the superior oblique.

As common activities are usual with the gaze below the horizontal, the eye to take up fixation will be generally the hypotropic one. Therefore, in a paresis of the superior rectus, the fixing eye will be commonly the one with the paretic muscle, with resulting increased innervation, in accordance with Hering's law, to the contralateral synergist (inferior oblique). On the other hand, in a paresis of the superior oblique, the affected eye remains in hypertropia and fixation is taken up by the other eye, so that the spasm of the ipsilateral antagonist will predominate, which also happens to be the inferior oblique. This explains the frequency of post-paretic spasms of the inferior oblique, and consequently the frequency of operations on this muscle.

Case 1, aged 14 yrs, came complaining of eyestrain. There was no error of refraction. A right hyperphoria of $6^{\Delta}$ was found with the Maddox rod, and of $4^{\Delta}$ with the Maddox wing test ; The synoptophore angle was $0^{\circ} \mathrm{R} / \mathrm{L} 5^{\Delta}$. 
Diplopia was apparent in laevoelevation. Fig. $1(c)$ indicated a right inferior oblique spasm. The Hess chart revealed a paresis of the left superior rectus with a spasm of the right inferior oblique.

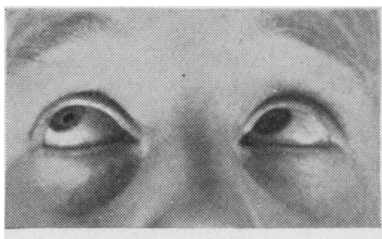

(a)

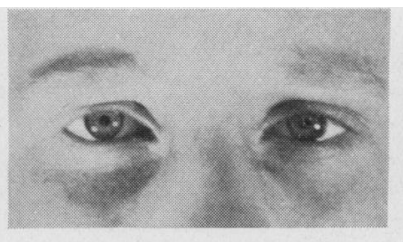

(b)

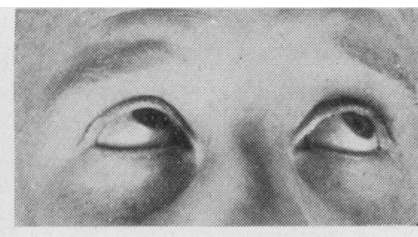

(c)

Fig. 1.-Case 1, paresis of left superior rectus.

Operation.-A marginal myotomy of the right inferior oblique, near its insertion, was performed in July, 1953.

A week later the Maddox rod and the Maddox wing test revealed a right hyperphoria reduced to $0.5^{\Delta}$, the Hess chart was normal, in the laevoelevation there was equal excursion of both eyes (Fig. 2 c), and the synoptophore angle was $0^{\circ} \mathrm{R} / \mathrm{L} 1^{\Delta}$.

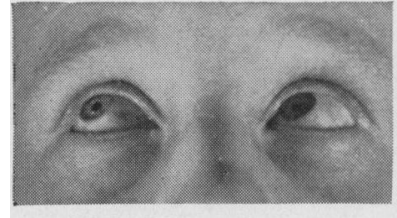

(a)

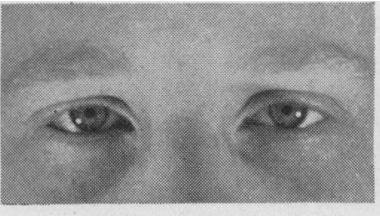

(b)

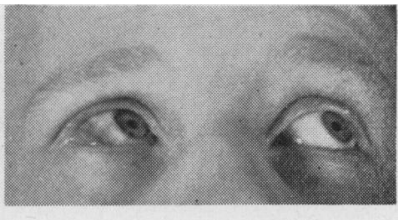

(c)

Fig. 2.-Case 1, after marginal myotomy of right inferior oblique.

Diagnosis-Paresis of left superior rectus.

Classification-Primary vertical squint of Group 1 (paresis of one elevator).

Case 2, aged 8 yrs, sought advice for ptosis of the right eye. A definite right hypotropia existed (Fig. $3 b$ ), with moderate amblyopia ex anopsia of that eye (20/80). The pseudoptosis vanished on fixing with the right eye (Fig. $3 \mathrm{c}$ ).

There was complete paralysis of the right superior rectus (Fig. $3 a$ ), inhibitional paresis of the left superior oblique (Fig. $3 d$ ), and relative overfunction of the left inferior oblique and right inferior rectus.

A moderate convergent squint was also apparent (Fig. $3 b$ and $c$ ), which disappeared on looking straight up and was more marked on looking straight down.

$$
\begin{aligned}
\text { Synoptophore: } & \text { Fixing right eye }+10^{\circ} \mathrm{L} / \mathrm{R} 20^{\circ} . \\
& \text { Fixing left eye }+10^{\circ} \mathrm{L} / \mathrm{R} 14^{\circ} . \\
& \text { Fusion at }+10^{\circ} \mathrm{L} / \mathrm{R} 14 \triangle .
\end{aligned}
$$

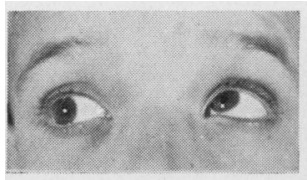

(a)

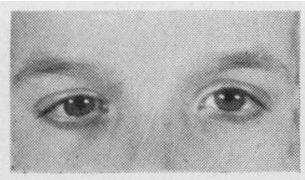

(b)

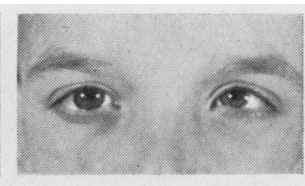

(c)

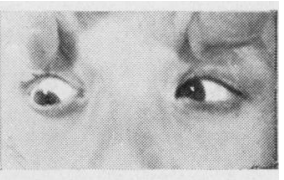

(d)

FIG. 3.-Case 2, paralysis of right superior rectus and slight convergent squint. 
Diagnosis.-Paralysis of right superior rectus, with added convergent squint of slight degree.

Operation.-Right inferior rectus recession $(5 \mathrm{~mm}$.) ; left inferior oblique myectomy (with excision of fragment of muscle $6 \mathrm{~mm}$. long) near its insertion ; left medial rectus recession $(5 \mathrm{~mm}$.).

The result was very good as the dextroelevation was completely normalized (Fig. 4 a), even though the paralysed superior rectus had not been touched, and the hypotropia and pseudo-ptosis of the right eye disappeared (Fig. $4 \mathrm{~b}$ ).
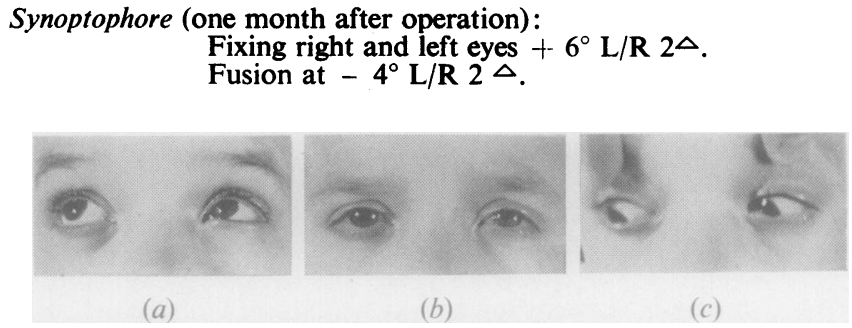

Fig. 4.-Case 2, after one-stage operation on both vertical and horizontal muscles.

Classification.-Primary vertical squint of Group 1 (paralysis of one elevator), with added convergent squint of slight degree.

Case 3, aged 6 yrs, presented with alternating divergent stabismus since the age of $1 \mathrm{yr}$. Examination revealed, in addition to the divergent strabismus, a definite hypertropia of the right eye (Fig. 5), with paresis of the left superior rectus and a slight true left ptosis.

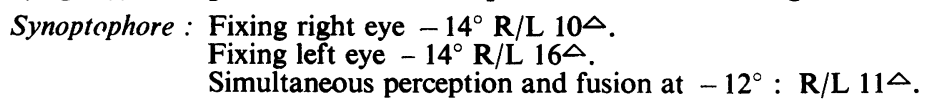

Diagnosis.-Paralysis of left superior rectus, concomitant divergent strabismus.

Operation.-Horizontal and vertical muscles operated simultaneously: Left lateral rectus recession $(5 \mathrm{~mm}$.) ; left medial rectus resection $(8 \mathrm{~mm}$.) ; left inferior rectus recession $(5 \mathrm{~mm}$.) ; marginal myotomy of right inferior oblique.

The result was satisfactory (Fig. $6 a$ ) with normalization of the laevoelevation (Fig. $6 b)$; the slight left ptosis persisted. Ten months later the patient had simultaneous perception and fusion at $0^{\circ} \odot$.

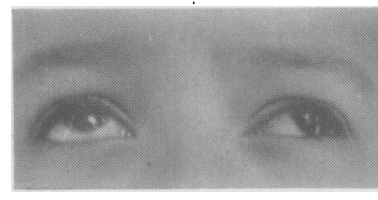

Fig. 5.-Case 3, paralysis of left superior rectus and divergent squint.

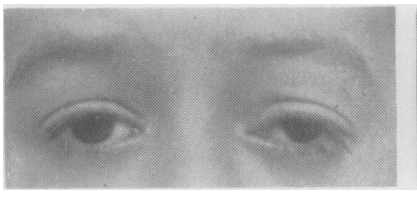

(a)

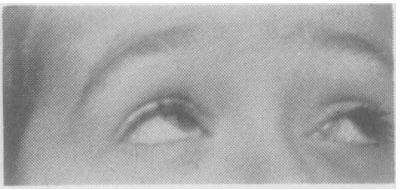

(b)

Fig. 6.-Case 3, after one-stage operation on both vertical and horizontal muscles.

Classification.-Primary vertical squint of Group 1 (paralysis of one elevator), with added concomitant divergent squint.

(ii) The inferior oblique and inferior rectus muscles are less frequently affected. In paresis of the inferior oblique muscle the affected eye remains in hypotropia and thus will generally take up fixation, with the result that supplementary innervation will also arrive to its contralateral synergist (superior rectus). In paresis of the in- 
ferior rectus muscle the eye remains in hypertropia and fixation will generally be taken up by the other eye, so that the spasm of the ipsilateral antagonist (also the superior rectus) will predominate. This is why in such cases one frequently has to do a retroplacement or marginal myotomy of the spasmodic superior rectus, surgery on the superior oblique being more rarely necessary.

Case 4, aged 11 yrs, presented with a convergent strabismus of the right eye from the age of 2 yrs. There was a slight unilateral myopia $(-0.75)$ and also an amblyopia ex anopsia of 20/80 p. of this eye; this improved to 20/50 after 2 months of permanent occlusion of the left eye.

Apart from a marked convergent squint of the right eye, which did not vary on looking upward or downward, a paresis of the right inferior oblique was apparent in laevoelevation.

$$
\begin{array}{ll}
\text { Synoptophore : Fixing right eye }+24^{\circ} \mathrm{L} / \mathrm{R} 6^{\circ} . \\
& \text { Fixing left eye }+22^{\circ} \mathrm{L} / \mathrm{R} 4 \Delta .
\end{array}
$$

Abnormal retinal correspondence (images crossing at $+5^{\circ}$ ). A vertical deviation was also appreciated on subjective examination $\left(L / R 4^{\triangle}\right)$.

Diagnosis.-Paresis of right inferior oblique, concomitant convergent squint.

Operation.-Right medial rectus recession $(5 \mathrm{~mm}$.) ; right lateral rectus resection ( $8 \mathrm{~mm}$.) ; marginal myotomy of left superior rectus.

After 3 months the cover test was negative and laevoelevation was normal.

Synoptophore : $0^{\circ} \theta$.

Fusion only with oscillation.

Classification.-Primary vertical squint of Group 1 (paresis of the right inferior oblique), with concomitant convergent squint.

\section{Group 2. Paresis of Both Elevators or Depressors of One Eye}

The paresis of both elevators (superior rectus and inferior oblique) of one eye is accompanied by pseudo-ptosis which is generally the reason for consultation. The false ptosis becomes evident on making the cover test, since when the hypotropic eye is obliged to fix the ptosis automatically disappears.

Case 5, aged 20 yrs, underwent two operations 4 years ago for marked convergent squint of the left eye.

In September, 1952, she complained of ptosis of the left eye. There was a residual left convergent squint of about $10^{\circ}$ (with amblyopia ex anopsia of $2 / 30$ ptly), and a pseudoptosis due to paresis of both elevators of that eye (Fig. $7 a, b, c)$. Dextro- and laevodepressions were normal.

Synoptophore : Fixing right eye $+10^{\circ} \mathrm{R} / \mathrm{L} 26 \Delta$

Fixing left eye $+10^{\circ} \mathrm{R} / \mathrm{L} 30^{\Delta}$.

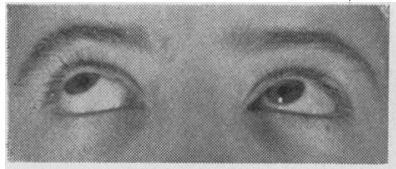

(a)

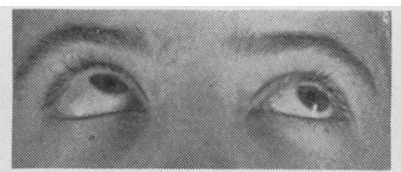

(b)

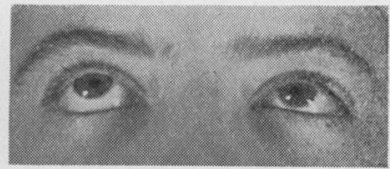

(c)

FIG. 7.- Case 5, paresis of both elevators (inferior oblique and superior rectus) of left eye, and residual convergent squint.

Diagnosis.-Paresis of superior rectus and inferior oblique of left eye ; moderate residual convergent squint.

Operation.-Even though the right eye was fixing there was no spasm of the ipsilateral antagonists (normal laevo- and dextrodepression), and it was therefore decided to operate 
on the contralateral synergists : right superior rectus for the paretic left inferior oblique (Fig. $7 a$ ), and right inferior oblique for the paretic left superior rectus (Fig. $7 b$ ). In October, 1952, a right superior rectus recession $(5 \mathrm{~mm}$.) and a myectomy of the right inferior oblique were performed simultaneously.

The result was the disappearance of the pseudo-ptosis and an almost complete normalization of ocular motility (Fig. $8 a, b, c$ ). The residual convergent squint persisted without variation (Fig. $8 c$ ) as no operation was done on the horizontal muscles.

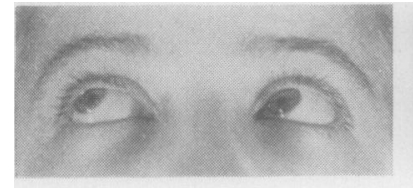

(a)

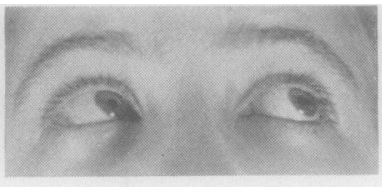

(b)

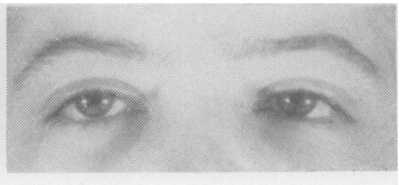

(c)

FIG. 8.-Case 5, after operation on vertical muscles only of right eye.

Classification.-Primary vertical squint of Group 2 (paresis of both elevators of one eye), with added horizontal (convergent) squint.

The paresis of both depressors of an eye (inferior rectus and superior oblique) is relatively rare, even though in Scobee's series, mentioned above, it was somewhat more frequent than that of both elevators (twenty cases against six).

Group 3. Bilateral Paresis (OR Spasm) of Twin Muscles.-In this group are included the relatively frequent cases with paresis of the homologous muscles of each eye (e.g. both superior obliques).

(i) Equal Paresis of Both Muscles.-There is no vertical deviation in the primary position, as both eyes take the same relative level. Binocular single vision can therefore continue to be exercised in the primary position, and a horizontal deviation may not be added.

Case 6, aged 8 yrs, presented from birth a frank bilateral hypertropia in adduction (Fig. $9 c$ and $e$ ), with binocular fixation in the primary position (Fig. $9 d$ ). Study of ocular motility showed a frank paralysis of both superior oblique muscles (Fig. $9 f$ and $g$ ), with inhibitional paresis of both superior recti (Fig. $9 a$ and $b$ ).

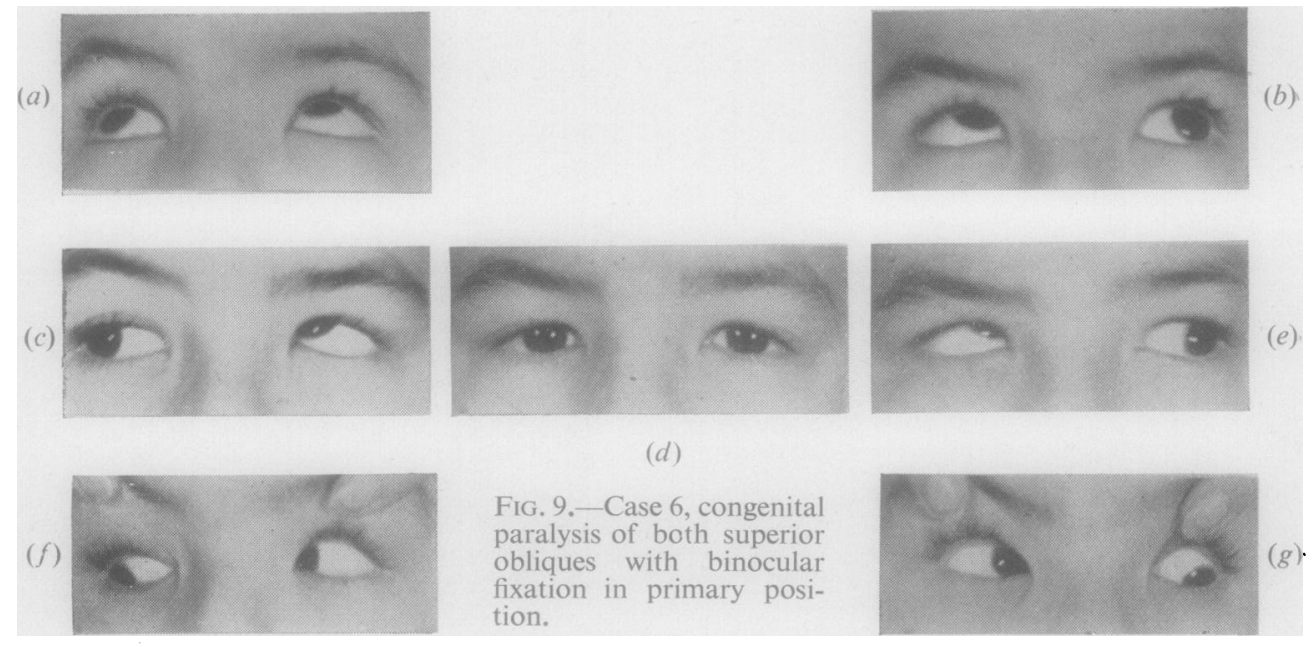


Synoptophore : Fixing right eye $+7^{\circ} \mathrm{L} / \mathrm{R} 22 \Delta$.

Fixing left eye $+7^{\circ} \mathrm{R} / \mathrm{L} 22 \Delta$.

Fusion and stereopsis at $0^{\circ} \theta$.

Diagnosis.-Paralysis of both superior obliques.

In our opinion, myectomy of both inferior obliques and retroplacement of both inferior recti (in one or two stages) would be the procedure indicated, but the parents do not wish an operation until the patient is older.

Classification.-Primary vertical squint of Group 3 (paralysis of twin muscles : superior obliques), without horizontal squint.

Case 7, aged 5 yrs, had from birth an unsightly bilateral elevation in adduction. There was binocular fixation, and the cover test in the primary position was negative (Fig. $10 \mathrm{~b}$ ). In dextro- and laevoelevation a marked spasm of both inferior obliques was noted (Fig. $10 a$ and $c$ ). Dextro- and laevodepression were practically normal.

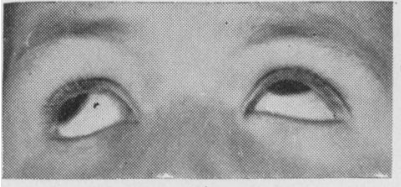

(a)

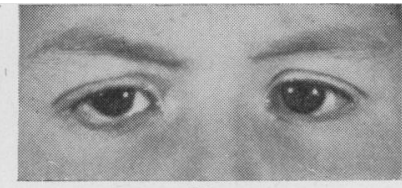

(b)

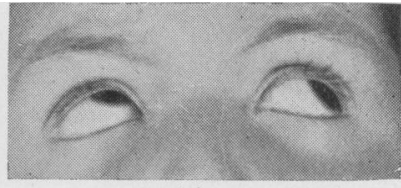

(c)

Fig. 10.-Case 7, marked spasm of both inferior obliques with binocular fixation in primary position.

Synoptophore : Fixing right eye $-2^{\circ} \bullet$.

Fixing left eye $-2^{\circ}$ R/L $2 \Delta$.

Fusion at $-2^{\circ}$ R/L $3^{\Delta}$.

Diagnosis.-Marked spasm of both inferior obliques: ? primary ? secondary to paresis of both superior recti.

Operation.-In 1952 a marginal myotomy of the right inferior oblique and a myectomy of the left inferior oblique were done. A week after the operation only a partial decrease of the spasm of the inferior obliques was noted (Fig. $11 a$ and $b$ ). As the spasm continued unchanged a further operation was performed 2 months later. The right inferior oblique appeared normal (without traces of the previous operation). In the left inferior oblique the stumps of the myectomy had re-adhered, and the muscle showed only a transverse scar in the site of the former operation. An extensive myectomy of both inferior obliques was performed, excising a fragment of muscle some $8 \mathrm{~mm}$. long.

(a)

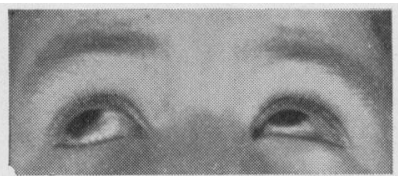

Fig. 11.-Case 7, after first operation, showing decrease in spasm of inferior obliques.

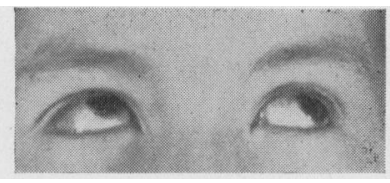

The result was fairly satisfactory, as only a slight spasm of the left inferior oblique (Fig. $12 a$ ) and a slight paresis of the right inferior oblique were noted (Fig. 12 c). In the primary position binocular fixation was maintained (Fig. $12 b$ ), but the cover test now showed a slight left hyperphoria.

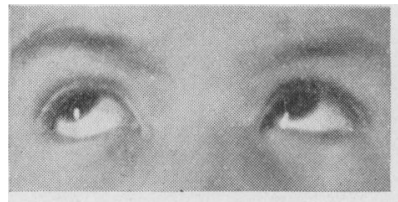

(a)

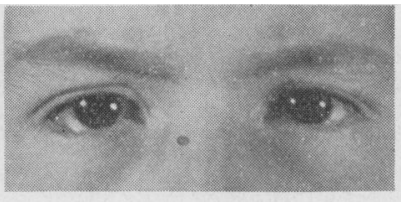

(b)

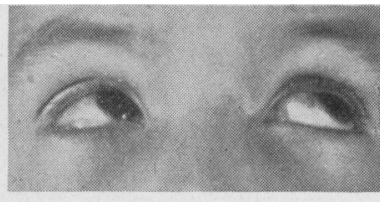

(c)

Fig. 12.-Case 7, after second operation, showing disappearance of spasm of inferior obliques. 
Synoptophore (15 days after operation) :

Fixing right eye $-3^{\circ} \mathrm{L} / \mathrm{R} 12^{\Delta}$.

Fixing left eye $-5^{\circ} \mathrm{L} / \mathrm{R}{ }^{\Delta} \Delta$

Fusion at $0^{\circ} \mathrm{L} / \mathrm{R} 5^{\circ}$.

There was no cyclophoria either before or after the operations.

Classification.-Primary vertical squint of Group 3 (paresis or spasm of twin muscles), without horizontal squint.

Comment.-This case raises the interesting problem of the existence of a "primary" bilateral spasm of the inferior oblique muscles without a previous paresis of the ipsilateral superior obliques or of the contralateral superior recti, and without a secondary overaction due to overflow of impulses from the medial recti in convergent spasmodic squint. Because of its characteristics the case seems to be one of primary spasm of the inferior obliques, though it is also possible that a paresis of both superior recti may have existed first, leaving spasm of the inferior oblique muscles after healing.

(ii) Greater Paresis of One Muscle.-A vertical deviation will be manifest in the primary position, and as binocular single vision is thus broken a horizontal deviation may be added.

Case 8, aged $3 \frac{1}{2}$ yrs, presented with alternating convergent squint from birth. Ocular motility was completely normal, and no spasm of the inferior obliques could be noticed. Alternating occlusion, which would probably have revealed the hidden vertical defect appearing on dissociation at the synoptophore, was not carried out.

$$
\begin{aligned}
\text { Synoptophore : } & \text { Fixing right eye }+25^{\circ} \mathrm{L} / \mathrm{R} 14 \triangle . \\
& \text { Fixing left eye }+25^{\circ} \text { R/L } 7 \Delta .
\end{aligned}
$$

Pre-operative Diagnosis.-Alternating convergent squint.

Operation.-Medial rectus recession $(5 \mathrm{~mm}$.) ; lateral rectus resection $(10 \mathrm{~mm}$.) in the left eye. The operation corrected the horizontal strabismus, and this result was maintained $2 \frac{1}{2}$ years after operation. However, the cover test showed a frank hypertropia of each eye from the first post-operative examination. The study of versions now showed a frank paresis of both superior recti, with the corresponding spasm of the inferior obliques.

\section{Synoptophore : Fixing right eye $0^{\circ} \mathrm{L} / \mathrm{R}$ more than $22 \Delta$.} Fixing left eye $+4^{\circ} \mathrm{R} / \mathrm{L} 12^{\triangle}$.

At times he could fuse at $0^{\circ}$, and at times he suppressed the image from one or the other eye (which was then found to be hypertropic).

Post-operative Diagnosis.-Paresis of both superior recti; concomitant convergent squint.

In our opinion a myotomy of both inferior obliques should be performed, but his parents do not desire a second operation.

Classification.-Primary vertical squint of Group 3 (unequal paresis of both superior recti), with added convergent squint.

Group 4. Mixed or Multiple Paresis.*-This group includes the rare cases of paresis in each eye of a different rectus muscle (e.g. the superior rectus of one eye and the inferior rectus of the other), or of a different oblique muscle (e.g. the superior oblique of one eye and the inferior oblique of the other). Scobee's series, mentioned above, contains eleven patients with paralysis of the superior rectus of one eye and of the inferior rectus of the other.

In this group should also be included cases of multiple paresis of elevators and depressors (e.g. inferior rectus and inferior oblique of one eye ; paralysis of the third cranial nerve; etc.).

- Patients with inhibitional palsy of a contralateral antagonist are not to be included in this group, as this is secondary to the paresis of a vertical muscle of the other eye (e.g. paresis of the right superior oblique with spasm of the left inferior rectus and inhibitional palsy of the left superior rectus). Such pationts must be included in Group 1 (See Case 2, Fig. $3 a$ and $d$ ). 
Group 5. CONCOMITANT HyPertropia (OR HyPerphoria).- The best known case in this group is that of simple hyperphoria, without horizontal deviation, which in the Hess chart shows an even vertical deviation in all directions of gaze.

It is also possible that a child with a latent hyperphoria may develop a convergent squint, like any other child, after a disease such as measles. In such cases a horizontal strabismus is associated with a concomitant hypertropia. The characteristics of this hypertropia are the invariability of the vertical angle of squint in the different directions of gaze, or upon changing fixation from one eye to the other.

We have at times seen patients with horizontal squint where the synoptophore measurements showed a moderate vertical component, but in which a careful study of the ocular motility showed no noticeable muscle paresis. After correcting the horizontal squint the vertical concomitant deviation persisted, and a second operation had to be performed to correct the hypertropia.

Case 9, aged 8 yrs, presented with alternating convergent squint from the age of 2 yrs. The cover test also showed a hypertropia of the right eye upon fixation with the left eye and hypotropia of the left eye upon fixation with the right eye. No noticeable muscle paresis or spasm could be demonstrated.

Synoptophore : Fixing right eye $+22^{\circ} \mathrm{R} / \mathrm{L}_{4} \Delta$.

Fixing left eye $+22^{\circ} \mathrm{R} / \mathrm{L}^{\Delta} \Delta$.

Abnormal retinal correspondence with images crossing at 0 . On subjective examination a vertical deviation $\left(\mathrm{R} / \mathrm{L} 4^{\Delta}\right)$ was also noted.

Operation.-As no muscle could be held responsible for the hypertropia, which was nevertheless very evident, it was decided to take advantage of the operation on the horizontal muscles of the right eye to perform a marginal myotomy of the inferior oblique of this eye (higher eye).

In January 1951 a medial rectus recession $(5 \mathrm{~mm}$.), lateral rectus resection $(8 \mathrm{~mm}$.), and marginal myotomy of the inferior oblique of the right eye were performed. The convergent squint disappeared and, although a slight paresis of the myotomized right inferior oblique was now noticeable, the hypertropia of the right eye persisted.

Synoptophore : Fixing right and left eyes $0^{\circ} \mathrm{R} / \mathrm{L}^{\Delta}$.

Subjective vertical deviation: $\mathrm{R} / \mathrm{L} 4^{\Delta}$.

Glasses.-Prism glasses were prescribed : $2^{\Delta}$ base down in front of the right eye and $2^{\Delta}$ base up in front of the left eye.

Follow-up.-After one year the concomitant hypertropia of the right eye persisted. On the synoptophore the subjective vertical deviation was of $R / L 7^{\Delta}$, and there was fusion only with oscillation.

Operation.-Left inferior rectus recession $(4 \mathrm{~mm}$.) (hypotropic eye). The result was the disappearance of the vertical deviation in the primary position. A moderate paresis of the recessed left inferior rectus was now evident.

Fusion and stereopsis were developed with exercises on the stereoscope.

Up to $2 \frac{1}{2}$ years since the second operation the cover test showed no deviation in the primary position, there was a slight paresis of the operated vertical muscles (right inferior oblique and left inferior rectus), and in the synoptophore there was fusion at $0^{\circ}$, with fusion amplitude from $-5^{\circ}$ to $+10^{\circ}$.

Classification.-Primary vertical strabismus of Group 5 (concomitant hypertropia), with added convergent squint.

In patients with concomitant hypertropia we should now consider (in view of the experience gathered with Case 8) that a recession of the superior rectus of the 
hypertropic eye would be the procedure of choice, so as not to interfere with the depressor muscles of greater functional importance.

Group 6. Dissociated Vertical Divergence.-When this type of defect is present with no horizontal deviation, it is known as anaphoria, alternating hyperphoria, or double hyperphoria. The fundamental characteristic in such cases is that there is usually binocular fixation (bifixation), so that the double hyperphoria is only apparent with occlusion of one or the other eye.

This anomaly is sometimes accompanied by permanent horizontal squint, when it is known as anatropia, alternating hypertropia, or double hypertropia.

Verhoeff (1941), in a total of 42 patients with occlusion hypertropia, as he called it, found 24 cases associated with a permanent strabismus (seventeen of which were cases of esotropia), and eighteen cases with bifixation, that is, without a permanent horizontal strabismus.

The nature of dissociated vertical divergence has been widely disputed. There seems to exist an aberration in the postural tonus of the muscular apparatus that adjusts the position of the eyes in a vertical direction (Posner, 1952). The upward movement of the non-fixing eye (under cover in a case of anaphoria, or squinting in one of anatropia) is probably effected by the normal reflex route of Bell's phenomenon (Schlossman, 1952).

Some confusion is apparent in the literature regarding the existence or nonexistence of overfunction of the inferior obliques in cases of dissociated vertical divergence.

Duke-Elder (1949) recognizes that the absence of spasm of the inferior obliques is a sign of diagnostic importance in dissociated vertical divergence when he states :

Nor does a change in the direction of the gaze horizontally alter the vertical deviation as it does in a spastic squint (due to overaction of the inferior oblique).

Bielschowsky (1938) is not quite definite on this point. On one hand he favours myectomy of the inferior obliques in cases of spasm of these muscles (Group 3 of his classification), and disapproves of operation on the vertical muscles in dissociated vertical divergence (Group 4 of his classification); on the other hand he uses photographs of the same child (Figs 3 and 6 in his paper) as examples both of a convergent squint with a marked bilateral spasm of the inferior obliques and of a convergent squint with double hypertropia.

Verhoeff (1941) states:

Such a condition, if bilateral, would be manifested during binocular vison (e.g. when neither eye was covered) by evidence of so-called overaction of the inferior obliques. Unfortunately, in my series of cases I did not investigate this question with sufficient care. However, in some of the cases this condition undoubtedly existed, and it is my impression, based on all the cases I have encountered, that overaction of the inferior obliques is common in cases of occlusion hypertropia.

Schlossman (1952) refers indirectly to this point when he says:

When fixation is alternately broken up by the cover test there is an overaction of the obliques, but this pattern is loose and shows the same variability as that of the horizontal muscles.

Our findings agree with those of Duke-Elder, as we believe that an important differential sign, which allows for the classification of a case in the group of dissociated vertical divergence, is to find normal ocular motility without spasm of the 
inferior obliques. In cases of double hypertropia with added convergent squint (cf. Case 10) the frank hypertropia of the non-fixing eye in the primary position is in contrast with the slight or non-existent hypertropia in the oblique gazes (Fig. 13).

However, it is necessary to explain a point which might lead to confusion. When the nose comes in the way of the eye in extreme adduction it automatically effects the occlusion of that eye (nasal cover test) thus inducing its hypertropia, and feigning a spasm of the inferior oblique. This was clearly observed in Case 10 after surgical correction of the horizontal deviation. There was no manifest spasm of the inferior obliques while it was possible to maintain binocular fixation. When the corneal reflection of the torch disappeared through the intervention of the nose, this eye showed a moderate hypertropia (Fig. 16, overleaf).

Before the operation for convergent squint, the study of versions in this patient generally showed an equal excursion of both eyes, but in a few instances a moderate hypertropia was seen in extreme adduction. The nose could not act as a dissociating element as dissociation already existed on account of the convergent squint. The hypertropia could then be attributed to the darkening of the adducted eye by intervention of the nose.

In patients with dissociated vertical divergence, if the illumination before one eye is gradually diminished (with a smoked glass wedge), this eye will gradually deviate upwards (Verhoeff, 1941). This is not to be confused with Bielschowsky's phenomenon, which is not constant, wherein the eye in hypertropia behind the screen will deviate downward when the smoked wedge is put in front of the other eye.

Case 10, aged 10 yrs, had squinted since birth ; delivery had been normal. Examination revealed alternating convergent squint with frank hypertropia of the non-fixing eye (Fig. $13 c$ and $d$ ). The study of versions usually showed an equal excursion of both eyes (Fig. $13 a, b, e, f$ ), but, at times, a moderate hypertropia was noted in the extreme adduction of each eye, when vision was obscured by intervention of the nose.

Synoptophore : Fixing right eye $+22^{\circ} \mathrm{L} / \mathrm{R}$ over $22 \triangle$.

Fixing left eye $+22^{\circ} \mathrm{R} / \mathrm{L}$ over $22^{\triangle}$.

On subjective examination (fixation with both eyes) there was equal height of both images, and fusion was attained at $+22^{\circ}$ with oscillation.

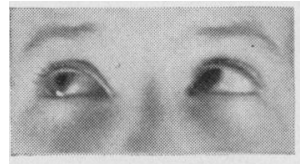

(a)

(e)

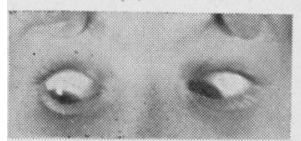

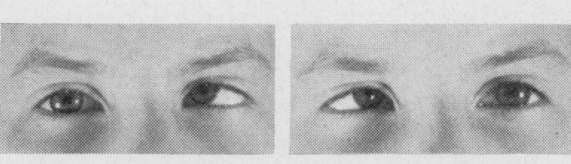

(c)

(d)

Fig. 13.-Case 10, double hypertropia and convergent squint.

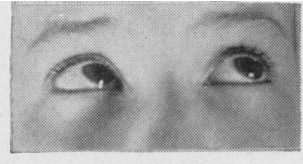

(b)
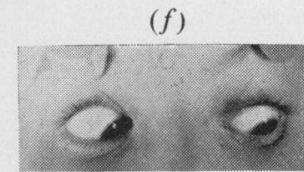

Diagnosis.-Double hypertropia, concomitant convergent squint.

Operation.-Medial rectus recession $(5 \mathrm{~mm}$.) and lateral rectus resection $(8 \mathrm{~mm}$.) in the 
left eye. The horizontal squint disappeared completely (Fig. $14 \mathrm{c}$ ), and the double hypertropia became a double hyperphoria (Fig. $15 a$ and $b$ ). Fusion rapidly developed with exercises on the stereoscope. The ocular movements were normal (Fig. $14 a, b, d, e$ ), save for a slight elevation of the adducted eye on intervention of the nose (Fig. $16 a$ and $b$ ).

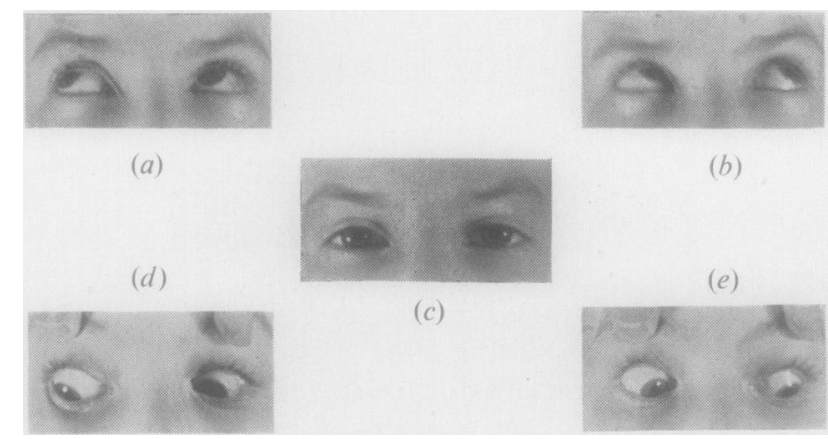

FIG. 14.-Case 10, after correction of horizontal deviation.

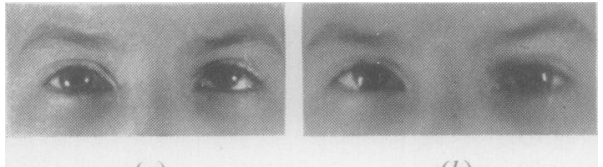

(a)

Fig. 15.-Case 10, double hyperphoria after correction of horizontal deviation.

(a) Left eye elevated after occlusion.

(b) Right eye elevated after occlusion.

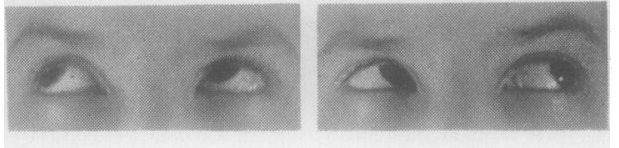

(a)

(b)

Fig. 16.-Case 10, the nose acting as an occluder provokes hyperphoria in the adducted eye (false spasm of inferior obliques).

\section{Synoptophore : Fixing right eye $+2^{\circ} \mathrm{L} / \mathrm{R}$ over $22 \Delta$ \\ Fixing left eye $-3^{\circ} \mathrm{R} / \mathrm{L}$ over $22^{\triangle}$. \\ Fusion with amplitude of $12^{\circ}$.}

Classification.-Primary vertical squint of Group 6 (dissociated vertical divergence), with added concomitant convergent squint.

\section{(B) Secondary Vertical Squint-Urist's Syndromes}

The tendency of an eye in permanent convergent strabismus to develop a secondary hypertropia is well known (strabismus convergens surso-adductorius).

The explanation generally given is that the adduction position entails some mechanical advantage to the inferior oblique in relation with its antagonist, the superior oblique. Scobee (1947) gives the following description to explain what he designates " normal " overaction of the inferior oblique:

The speed with which a muscle's point of insertion will move is proportional to the length of the muscle. The inferior oblique is longer $(37 \mathrm{~mm}$.) than the effective portion of the superior oblique $(20 \mathrm{~mm}$.), and the additional fact that the insertion of the inferior oblique is such that its arc of contact is longer than that of the superior oblique means that, with the same amount of contraction, the inferior oblique will produce greater vertical displacement, that is, elevation, than will the superior oblique, that is, depression. It also follows that, in maximum adduction of either 
eye, the inferior oblique is a better elevator than the superior oblique is a depressor ; this is because the inferior oblique makes a smaller angle with the visual axis than does the superior oblique by about $5^{\circ}$.

These arguments seem to contradict the well-known physiological superiority of the superior oblique over the inferior oblique, as it is a muscle constantly used in reading and other near work. Its importance is shown by its complicated anatomy and its innervation by a special cranial nerve.

Guibor $(1949,1950)$ uses the term "synkinetic overaction of the inferior oblique ", as he attributes the frequency of secondary spasm of this muscle to a spread of impulses through the oculomotor complex. Hypertonicity of convergence is associated with an overflow of impulses which pass to the inferior oblique directly through the third nucleus or indirectly through the medial longitudinal bundle. In this respect the anatomical neighbourhood of the nucleus of the inferior oblique with that of the medial rectus and Perlia's nucleus is interesting.

Spaeth (1948) also quotes the case of a patient with a left sixth-nerve palsy. The overaction of the contralateral synergist, the right medial rectus, was accompanied by a frank spasm of the right inferior oblique (Fig. 17 in his paper). This increased with attempted upward gaze to the left, even though the paralysis of the left external rectus prevented the left eye from rotating into the field of action of the left superior rectus. As Spaeth says :

In this case a true spasm was present in the inferior oblique muscle. It seems as if the overaction of the inferior oblique muscle must be connected with the simultaneous overaction of the right internal rectus muscle. This has been suggested before in considering overconvergence.

The following case demonstrates the causal relationship between overaction of the medial rectus and spasm of the inferior oblique.

Case 11, aged 2 yrs, presented with convergent squint after a febrile condition suspicious of frustrated infantile paralysis (the child staggered on walking). Examination, carried out a week after the squint was first noticed, showed convergence of some $20^{\circ}$ of the left eye with complete left lateral rectus paralysis. There was no spasm of the inferior oblique muscles. A year later there was only a slight limitation of the abduction of the left eye. The convergent squint practically disappeared on looking straight up and increased on looking straight down. A frank spasm of the left inferior oblique was now clear (Fig. $17 a)$.

Synoptophore : Fixing both eyes $+14^{\circ} \mathrm{L} / \mathrm{R} 4^{\triangle}$.

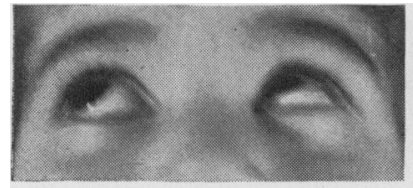

(a)

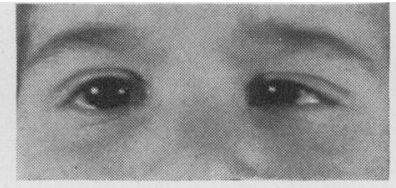

(b)

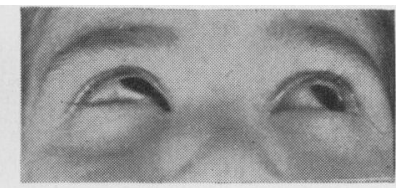

(c)

Fig. 17.-Case 11, secondary spasm of inferior obliques one year after left lateral rectus paralysis.

This spasm of the left inferior oblique was obviously secondary to the horizontal deviation, as a year previously (when the paralysis of the lateral rectus appeared) it did not exist. It is natural to relate it with the spasm of the left medial rectus. A slight 
spasm of the right inferior oblique was also noticeable. After occlusion of the right eye for 10 days this increased to the same degree as that in the left eye (Fig. 17 c). The increased spasm of the inferior oblique in the right eye was due to the fact that when the right eye was occluded the left eye with the paretic muscle took up fixation, and, in accordance with Hering's law, the compensatory innervation to the left lateral rectus brought about a spasm of the right medial rectus. As Guibor suggested, this greater innervation radiated to the inferior oblique nucleus of the same side.

Urist $(1951,1952)$ classified the various types of vertical deviation secondary to horizontal defects in well defined groups, and insisted on the diagnostic importance of the straight upward and downward gaze. For this reason, when referring to secondary vertical squints, we speak of "Urist's syndromes".

Urist's four groups are as follows:

Group 1. Esotropia with Bilateral Elevation in Adduction.-Esotropia greater when looking down than when looking up ; left hypertropia on looking towards the right, and greater up and to the right; right hypertropia on looking to the left, and greater up and to the left; good near point of convergence; vertical measurements suggest paresis of both superior recti with spasm of both inferior obliques. As the underlying condition seems to be a hypertonicity of convergence, a bilateral medial rectus recession can correct both the horizontal strabismus and the secondary vertical deviations.

Case 12, aged 5 yrs, presented with convergent squint of the right eye from the age of 8 months. Examination showed a convergent strabismus of the right eye of about $20^{\circ}$, with marked amblyopia ex anopsia (1/40), frank paresis of the left superior rectus, and spasm of the right inferior oblique (amblyopic eye). There was no noticeable spasm of the left inferior oblique. After occlusion of the left eye for 2 months vision increased in the right eye to 5/50, and a marked spasm was then evident in both inferior obliques. Five months after occlusion the visual progress stopped at 5/30. The patient presented all the characteristics of Urist's Group 1 (Fig. 18).

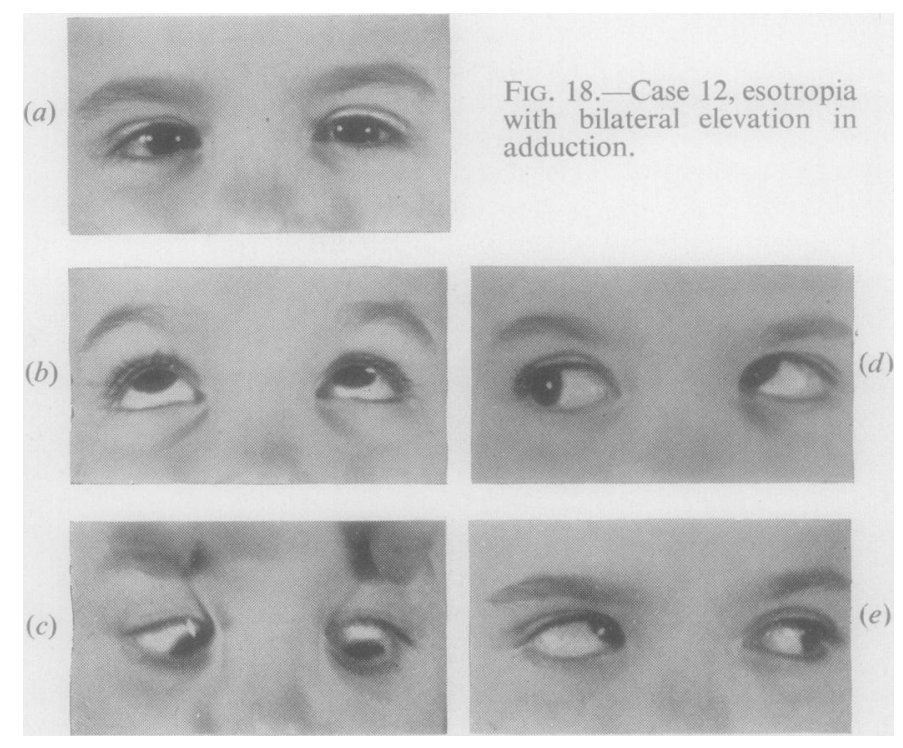


Operation. $-5 \mathrm{~mm}$. recession in both medial recti. A month after operation (Fig. 19) a decrease in the secondary spasm of the inferior obliques could be noted.

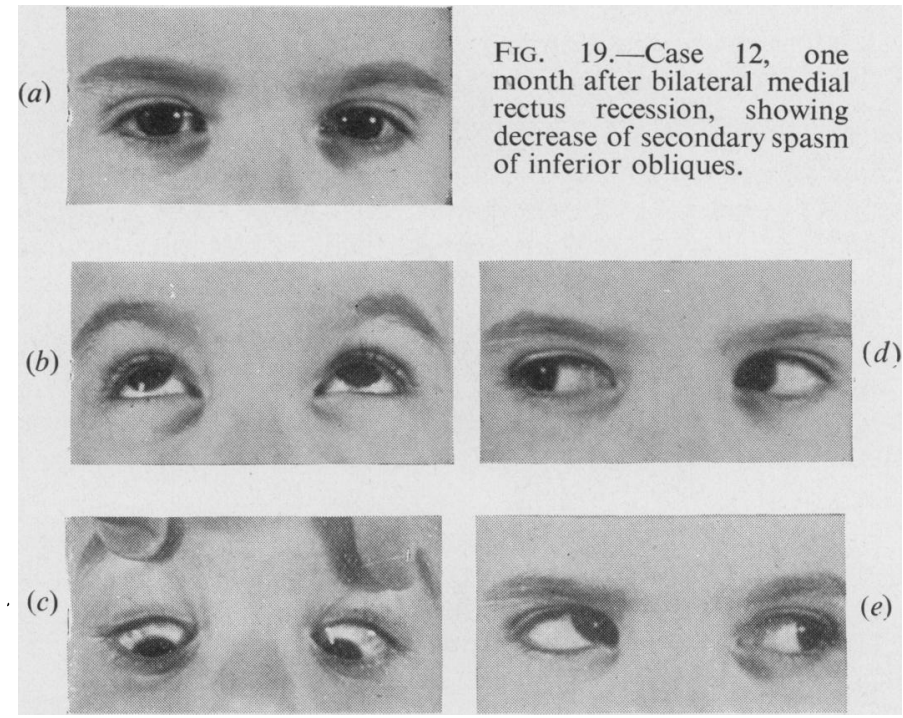

After 3 months the elevation on adduction disappeared (Fig. 20).

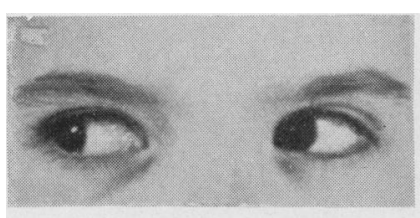

(a)

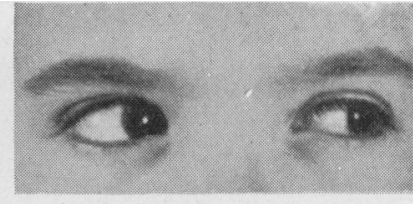

(b)

Fig. 20.-Case 12, 3 months after operation, showing disappearance of secondary spasm of inferior obliques.

Classification.-Secondary vertical squint of Group 1 (esotropia with bilateral elevation in adduction).

Comment.-Only the spasm of the inferior oblique in the non-fixing (amblyopic) eye was apparent at the start ; this might have led to the error of classifying the case as a primary vertical squint (paresis of the left superior rectus). Prolonged occlusion allowed for a correct classification.

Group 2. Esotropia with Bilateral Depression in AdDUCTION.-Greater esotropia looking up than down; depression of the right eye on looking to the left ; depression of the left eye on looking to the right; near point of convergence: 30 to $90 \mathrm{~mm}$.; vertical measurements suggest paresis of both inferior obliques with spasm of both superior recti ; resection of both underacting lateral recti indicated.

Case 13, aged 32 yrs, presented with convergent strabismus of the right eye since childhood, and marked amblyopia ex anopsia (1/50 ptly). Examination showed a right esotropia of $20^{\circ}$ on looking straight ahead. Vertical versions showed $30^{\circ}$ esotropia on looking up and $5^{\circ}$ on looking down. Lateral versions showed frank bilateral depression in adduction. The patient did not desire surgery. 
Classification.-Secondary vertical squint of Group 2 (esotropia with bilateral depression in adduction).

Group 3. Exotropia with Bilateral Elevation in AdDUCtion.-Greater exotropia when looking up than down; elevation of the right eye in looking to the left, and of the left eye in looking to the right ; good near point of convergence.

Case 14, aged 17 yrs, presented with divergent strabismus from childhood, and moderate amblyopia ex anopsia of the left eye $(5 / 20)$. Examination showed a left exotropia of $15^{\circ}$ on looking straight ahead. Vertical versions showed $30^{\circ}$ of exotropia on looking up and $5^{\circ}$ on looking down. Lateral versions showed bilateral elevation in adduction, which was greater in the left amblyopic eye (the more dissociated eye).

\section{Synoptophore : Fixing right eye $-22^{\circ} \mathrm{L} / \mathrm{R} 9 \Delta$. Fixing left eye $-22^{\circ} \mathrm{R} / \mathrm{L} 3 \triangle$. Fusion at $-22^{\circ} \mathrm{L} / \mathrm{R} 4 \Delta$.}

Operation.-Recession of both lateral recti $(6 \mathrm{~mm}$.). The immediate results were very poor, with only a slight decrease of the divergent squint, and no modification of the vertical deviations.

Comment.-Only after 6 months or one year will it be possible to judge the end-results. If the spasm of the inferior obliques then persists the patient should be classified in the group of mixed cases (see below), in which the reversible initial overfunction of the inferior obliques has gradually developed into an irreversible contracture.

Group 4. Exotropia with Bilateral Depression in Adduction.-Greater exotropia when looking down than up ; depression of each eye in adduction ; bad convergence ; resection of both medial recti indicated.

In connexion with these cases Urist insists on certain points which need be but briefly analysed.

The fact that the horizontal squint is noncomitant-with marked variation between upward and downward gaze-is of great diagnostic importance. This is fundamental in the recognition of Urist's syndromes. The secondary vertical deviation is a "dissociated hypertropia", as the hypertropia is clearly manifest in the position of extreme adduction when the nose intervenes between the light and the eye. On the other hand the vertical measurements are small in the primary position and when the lateroversion is not extreme.

The term " dissociated hypertropia", used by Urist, must be distinguished from " dissociated vertical divergence", the characteristics of which are quite different. As seen in Case 10, which presented convergent squint and dissociated vertical divergence, the double hypertropia is specially marked in the primary position (Fig. $13 c$ and $d$ ), and scanty or non-existent in the oblique positions. When the nose intervenes in the position of extreme adduction there is only a moderate hypertropia (pseudospasm of the inferior oblique).

Urist emphasizes that in secondary vertical deviations the eye that is more dissociated will show the greater, and at times the only, vertical deviation. The more dissociated eye may be either the amblyopic eye or the eye that has been occluded. The elevation in adduction will also be more pronounced in this eye.

In Cases 11 and 12 the spasm of the inferior oblique was noted first in the 
non-fixing eye only, and occlusion of the habitually fixing eye brought to notice the spasm in this other eye, showing that the condition was in fact bilateral.

Regarding the relative frequency of primary and secondary vertical deviations, Urist found in 615 patients with horizontal squint that 79 per cent. had a vertical component. Of these, 50 per cent. were included in one of his four groups, that is to say they had a secondary vertical deviation.

\section{METHOD OF Classification}

It is necessary to insist on postponing the final classification of any given case until after a more or less prolonged period of dissociation. In amblyopia ex anopsia the fixing eye will be occluded until vision is normal or there is no further visual progress. Thereafter, or if the squint is alternating, the occlusion will be transferred from one eye to the other (daily or every 2 days) during a period of not less than 15 days.

On various occasions an apparently pure horizontal squint has shown a marked paresis of a vertical muscle after occlusion.

Case 15, aged 4 yrs, presented with alternating convergent squint since birth. There had been obstetric trauma with a left brachial paralysis and ecchymosis of the left eye. Examination disclosed an alternating convergent squint of approximately $25^{\circ}$, without noticeable hypertropia and normal versions.

$$
\begin{gathered}
\text { Synoptophore : Fixing right eye }+29^{\circ} \mathrm{L} / \mathrm{R} \mathrm{14^{ \Delta }} \text {. } \\
\text { Fixing, left eye }+28^{\circ} \mathrm{R} / \mathrm{L} 14^{\Delta} . \\
\text { (double hyperphoria on the synoptophore) }
\end{gathered}
$$

The second examination with atropine cycloplegia showed the same objective findings, and the synoptophore readings were practically equal.

A dissociation period with alternate occlusion was then ordered and in the third examination, 14 days later, conditions were much changed.

Apart from the convergent squint the objective examination revealed a frank hypertropia of the right eye when fixing with the left eye, and a hypotropia of the left eye when fixing with the right eye. In dextroelevation a marked paresis of the left inferior oblique was now noted, and the synoptophore readings were also very different.

$$
\begin{gathered}
\text { Synoptophore : Fixing right eye }+30^{\circ} \mathrm{R} / \mathrm{L} 24 \triangle . \\
\text { Fixing left eye }+33^{\circ} \mathrm{R} / \mathrm{L} 26^{\circ} .
\end{gathered}
$$

At other times occlusion shows that an apparently unilateral vertical defect is really bilateral.

In Case 12 occlusion of the fixing eye demonstrated a synkinetic overaction of both inferior obliques, secondary to the horizontal squint.

Urist (1953) cited two cases of bilateral paralysis of the superior obliques in which, before surgery, only one paralysis was apparent with its corresponding torticollis and positive Bielschowsky's sign. Upon recession of the spasmodic inferior oblique the defect of that eye disappeared, and the paralysis of the other superior oblique, hidden until that moment, became apparent with a change of the torticollis to the other side. After a recession of the other inferior oblique a perfect result was obtained.

It seems possible that in other similar cases, no matter how obvious an apparent unilateral defect may appear, it may be possible to demonstrate a 
bilateral condition before surgery with a period of dissociation by alternate occlusion.

After dissociation the following procedure is followed in classifying particular cases. If we find a squint with marked vertical deviation and small or no horizontal deviation, the vertical deviation will be classified in the group of primary vertical squints.

The study of ocular versions in the four oblique diagnostic positions, while demonstrating one or more paresis of the vertical muscles, will permit the inclusion of the case in one of the four first groups of the classification.

If paresis is not apparent in any of the extra-ocular muscles it will be necessary to find out whether the case is one of concomitant hypertropia (Group 5) or of dissociated vertical divergence (Group 6).

Our findings are always checked three times before surgery is proposed : the first examination without and the second with atropine cycloplegia, and the third after dissociation by occlusion.

Cases wherein a vertical deviation is associated with a pronounced horizontal deviation are the most complicated. Two possibilities then exist :

(a) that the vertical defect is secondary to the horizontal strabismus (syndromes of Urist)

(b) that the vertical strabismus is the primary condition, to which was added (after binocular single vision was broken) a horizontal deviation.

As indicated by Urist the study of the variations of the horizontal angle of squint on looking straight up and down is of the greatest importance in deciding between these two possibilities.

The increase of the angle of squint on downward gaze is not, as it may be thought, common to all convergent squints. We have studied this matter in all our patients and have found that in ordinary concomitant squint (without secondary vertical deviations) either the degree of convergent strabismus is the same in looking up or down, or it increases only slightly when looking down.

On the other hand in Urist's syndromes of Group 1 (esotropia with bilateral elevation in adduction) there is frank decrease (or even disappearance) of the convergent squint in looking up, and a marked increase when looking down (Fig. 18). Therefore no diagnostic doubts exist in typical cases.

If in supraversion and infraversion the degree of horizontal strabismus shows only slight variation or none at all, we discard the possibility that the vertical deviation is secondary to the horizontal strabismus. The case will be one of primary vertical defect plus a horizontal deviation. It is then sufficient to analyse the vertical defect (mentally disregarding horizontal deviation), following the pattern just studied for a pure vertical squint, in order to place it in one of the six groups of primary vertical squints.

\section{Surgical Applications of the Classification}

It is of great importance to determine whether the vertical deviation is primary or secondary to the horizontal deviation. 
Secondary Vertical Deviation.-In these cases the horizontal squint should be dealt with first, without touching the vertical muscles. After correction of the horizontal defect, the secondary vertical deviation often gradually disappears (Figs. 18 and 20). As indicated by Urist, a hasty operation on the vertical muscles will provoke their paralysis, adding a paretic primary hypertropia to a hypertropia that was secondary to the horizontal deviation.

The disappearance of the secondary vertical deviation may take many months or even 1 or 2 years, as stated by Urist, and therefore no immediate results can be expected.

A long period of observation will indicate whether it is necessary to operate thereafter on the vertical muscles. When the synkinetic overaction of the inferior obliques has lasted a long time, an irreversible contracture may develop secondarily, requiring treatment by surgical lengthening of these muscles.

Primary Vertical Deviation.-If the vertical defect is of the primary type (Groups 1 to 4, muscular paresis), the vertical defect must be removed surgically together with the horizontal deviation.

Burian (1950) states that he has never seen a horizontal deviation disappear when the vertical deviation was surgically removed, if the horizontal deviation was relatively large. In our opinion even a small horizontal deviation, if concomitant and permanent, will remain unmodified on correcting the vertical deviation. Thus in Case 5 the convergent squint continued without variation as no surgery was performed on the horizontal muscles (Figs $7 c$ and $8 c$ ). In Case 2, on the other hand, where vertical and horizontal muscles were operated upon simultaneously, even though the horizontal deviation was unimportant in comparison with the vertical, a perfect result was obtained with one operation (Figs. $3 b$ and $4 b$ ).

The vertical deviation of the primary type does not disappear upon correction of the horizontal strabismus. For this reason we insist that both must be treated simultaneously (Cases 2, 3, and 4).

It is often repeated that if the vertical deviation is greater than the horizontal the vertical deviation must be tackled first, and vice versa. To apply a " quantitative" criterion does not seem very logical. It would be the same as if in a case of retinal detachment with two holes, one big one and one small, it was thought necessary to coagulate the larger one first. It is more important to apply a " qualitative" criterion.

If the vertical deviation, even though small, is of the primary type (moderate but definite paresis in a vertical muscle), it must be tackled together with the horizontal deviation, which was probably consecutive to the disturbance of binocular vision by the vertical defect. Once the horizontal deviation has been added and has become permanent, it will not disappear in spite of the correction of the causative vertical defect.

On the other hand, if the vertical deviation is secondary, even though large, it can disappear (if contractures have not been added) upon correction of the 
horizontal deviation (Case 12), and therefore should not be dealt with in the first operation.

In cases of dissociated vertical divergence, the vertical defect cannot be eliminated surgically. if present, the horizontal squint must be treated, as its correction will permit binocular vision while the vertical defect remains latent (Case 10).

In the association of horizontal squint with a probable concomitant hypertropia (Group 5), two operations could be considered, as there it is doubtful whether, after eliminating the horizontal deviation, a previously hidden vertical muscle paresis might become manifest. Thus, if the study of versions does not show a muscular paresis to explain the hypertropia, and the condition remains unchanged after dissociation by prolonged occlusion, the case should be classified temporarily as a concomitant hypertropia with added horizontal deviation (Case 9). If, after the horizontal squint has been eliminated, this situation persists, the diagnosis will be confirmed and a superior rectus recession of the hypertropic eye will be performed.

\section{(C) Mixed Cases}

Two different types of primary vertical squint may be combined in the same patient. For example, Bielschowsky (1938) mentions having seen cases with trochlear nerve paralysis complicated with dissociated vertical divergence which made the interpretation of the diplopia test quite difficult. Theoretically a muscular paresis can also appear in a patient with a previous concomitant hyperphoria.

Fortunately such cases of mixed primary vertical squints are very rare.

On the other hand the combination of secondary vertical deviations (syndromes of Urist) with primary vertical strabismus are not so infrequent.

Case 16, aged 5 yrs, presented with a right convergent squint since birth. He has been submitted to periodic occlusion of the left eye since the age of one year. After a period of alternate occlusion examination revealed a convergent squint of about $20^{\circ}$ in the primary position (Fig. $21 \mathrm{~b}$ ), $5^{\circ}$ looking up, and $30^{\circ}$ looking down. There was marked spasm of the left inferior oblique (Fig. $21 a$ ) and a moderate spasm of the right inferior oblique (Fig. 21c). Vision was 20/80 in the right eye and 20/50 in the left eye.

\section{Synoptophore : Fixing right eye $+30^{\circ} \mathrm{L} / \mathrm{R} 14 \triangle$. Fixing left eye $+30^{\circ} \bullet$.}

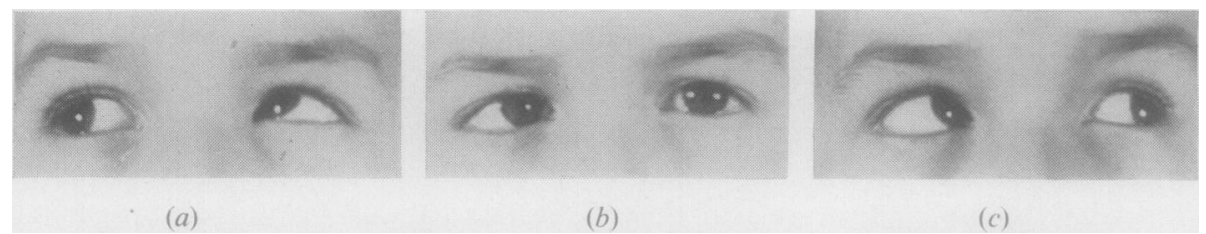

Fig. 21.-Case 16, unequal paresis of both superior recti, combined with Urist's syndrome Group 1.

Noncomitance of the convergent squint in looking up and down suggested the diagnosis of spasm of the medial recti (Urist's Group 1). The overaction of the inferior obliques could therefore be considered as secondary, but it was remarked that the greater spasm 
corresponded to the left eye, that is, to the fixing eye. We have seen before that in the first group of Urist's syndromes the elevation in adduction is greater in the more dissociated eye, that is, in the squinting eye.

A temporary diagnosis was made of a Urist's syndrome of Group 1 combined with a probable unequal paresis of both superior recti.

Operation.-Recession of $5 \mathrm{~mm}$. on both medial recti ; 6 months after operation a residual convergent squint of about $15^{\circ}$ remained both in the primary position (Fig. $22 b$ ) and when looking down. When looking up there was no horizontal deviation. A marked spasm persisted in the left inferior oblique (Fig. $22 a$ ), and a slight one in the right inferior oblique (Fig. $22 c$ ), but they were less marked than before the recession of both medial recti. Only part of the spasm of the obliques could, therefore, be considered secondary.

Synoptophore : Fixing right eye $+20^{\circ} \mathrm{L} / \mathrm{R}$ over $20^{\triangle}$.

Fixing left eye $+17^{\circ}$ R/L $: 4^{\triangle}$.

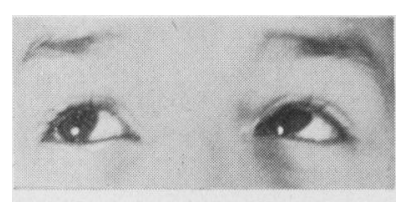

(a)

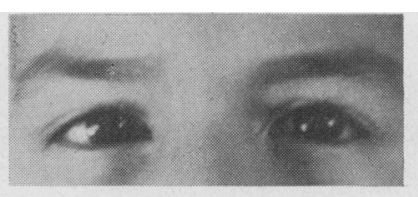

(b)

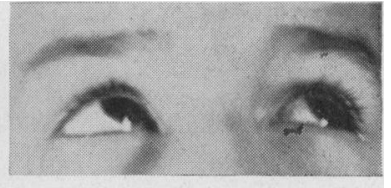

(c)

Fig. 22.-Case 16, after recession of both medial recti.

Diagnosis.-Unequal paresis of the two superior recti.

Operation.-Right lateral rectus resection $(10 \mathrm{~mm}$.) ; myectomy of left inferior oblique ; marginal myotomy of right inferior oblique. The result can be seen in Fig. $23(a, b, c)$; 9 months after the second operation the cover test was negative when looking forward, up, and down, and all versions were normal.

$$
\begin{aligned}
\text { Synoptophore : } & \text { Fixing right eye }+4^{\circ} \mathrm{L} / \mathrm{R} 14 \Delta . \\
& \text { Fixing left eye }+4^{\circ} \mathrm{R} / \mathrm{L} 2 \Delta .
\end{aligned}
$$

Intermittent fusion; at times suppresses right eye.

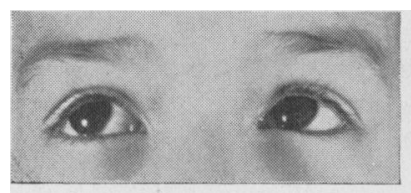

(a)

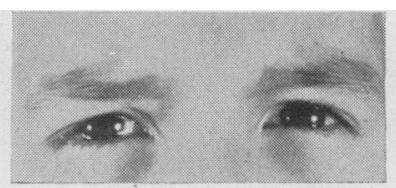

(b)

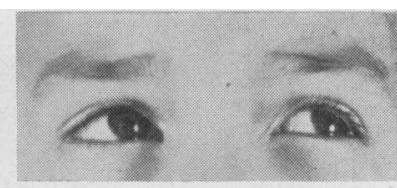

(c)

FIG. 23.-Case 16, after right lateral rectus resection and myotomy of both inferior obliques.

Classification.-Primary vertical squint of Group 3 (paresis of both superior recti) combined with esotropia with bilateral elevation in adduction (Urist's Group 1).

Another type of mixed vertical defect, not infrequently seen, is the addition of a secondary contracture of the inferior oblique when its synkinetic overaction has lasted too long. Guibor (1949) writes in this connexion:

If, however, the synkinetic overaction of the inferior oblique muscle persists over a period of several years, a contracture of this muscle tissue occurs, and the superior oblique relaxes ... It is important to stress that it is extremely difficult in such cases to differentiate between a superior rectus paralysis of the contralateral eye, a superior oblique paralysis of the ipsilateral, and an overaction of an inferior oblique resulting from a supranuclear disturbance (synkinetic overaction). 
Thus, in such cases, the secondary spasm of the obliques (Urist's Group 1) that at the beginning was functional and therefore reversible, gradually turns into an irreversible anatomical defect. A bilateral medial rectus recession will only partially decrease the elevation in adduction, since a real vertical defect now exists which may be classified as a primary vertical deviation. Therefore it will be necessary to elongate the contracted inferior obliques.

These cases cannot be differentiated from the primary paresis of two twin muscles (e.g. superior rectus or superior obliques), as there now exists an inhibitional palsy consecutive to the prolonged spasm of the inferior obliques.

\section{SUMMARY}

A practical classification of squints with a vertical deviation for surgical purposes is presented.

(A) Primary Vertical Strabismus (with or without added horizontal deviation):

(1) Paresis of an elevator or depressor muscle of one eye.

(2) Paresis of both elevators or depressor muscles of one eye.

(3) Bilateral paresis (or spasm) of twin muscles.

(4) Mixed or multiple paresis.

(5) Concomitant hypertropia (or phoria).

(6) Dissociated vertical divergence.

(B) Secondary Vertical Strabismus (Urist's syndromes):

(1) Esotropia with bilateral elevation in adduction.

(2) Esotropia with bilateral depression in adduction.

(3) Exotropia with bilateral elevation in adduction.

(4) Exotropia with bilateral depression in adduction.

(C) Mixed Cases.-The method to be followed in classifying particular cases, and the surgical application of the classification are analysed. It is emphasized that a period of dissociation by occlusion is always necessary before a final classification is made.

\section{REFERENCES}

Bielschowsky, A. (1938). Arch. Ophthal. (Chicago), 20, 175.

Burian, H. M. (1950). Amer. J. Ophthal., 33, 577.

DUKE-ELDER, S. (1949). "Text-book of Ophthalmology”, vol. 4, p. 4179. Kimpton, London. DunNington; J. H.. and RegAN, E. F. (1950). Arch. Ophthal. (Chicago), 44, 813.

Guibor, G. P. (1949). Amer. J. Ophthal., 32, 221. (1950). In “Strabismus Ophthalmic Symposium”, ed. J. H. Allen, p. 272. Mosby, St. Louis.

Posner, A. (1952). Personal communication tó Schlossman' (1952).

Schlossman, A. (1952). Amer. J. Ophthal., 35, 795.

SCOBEe, R. G. (1947). “ The Oculorotary Muscles”, p. 289. Kimpton, London.

(1951). Amer. J. Ophthal., 34, 817.

SPAETH, E. B. (1948). Ibid., 31, 1553.

URIST, M. J. (1951). Arch. Ophthal. (Chicago), 46, 245. (1952). Ibid., 47, 220.

- (1953). Ibid., 49, 382.

VERHOEFF, F. H. (1941). Ibid., 25, 780.

WhITE, J. W., and BRown, H. W. (1939). Ibid., 21, 999. 